\title{
DESDIFERENCIAÇÃO DO CÂNCER DA PRÓSTATA APÓS TERAPIA ANTIANDROGÊNICA
}

\author{
Rogério Moritz, Miguel Srougi*, Valdemar Ortiz, Kátia Ramos Moreira leite, luciano Nesrallah, \\ Marcos Dall'Oglio, Alexandre Crippa Sant'Anna \\ Trabalho realizado na disciplina de Urologia, Universidade Federal de São Paulo, São Paulo, SP.
}

RESUMO - ОвјETIVo. 0 bloqueio androgênico neo-adjuvante em câncer da próstata produz involução do volume tumoral sem meIhorar a evolução desses pacientes. Uma das explicações para esse fenômeno é a aquisição de comportamento mais agressivo pelas células tumorais remanescentes que, morfologicamente, apresentam aspecto mais indiferenciado após o bloqueio androgênico. Os objetivos do presente estudo foram avaliar a freqüência de desdiferenciação celular após tratamento antiandrogênico e definir se a neoplasia remanescente apresenta sinais de maior agressividade biológica.

Métodos. Trinta pacientes portadores de câncer da próstata localmente avançado foram submetidos a tratamento antiandrogênico neoadjuvante por quatro meses, seguido de prostatectomia radical. Foram comparados os escores de Gleason da biópsia e do espécime cirúrgico. Ademais, mediu-se $o$ índice de proliferação celular, determinado por imunohistoquímica para o PCNA, sendo considerados positivos os testes com reação nuclear intensa. A porcentagem de núcleos positivos, determinada em 500 células, foi confrontada com as diversas categorias do escore de Gleason do espécime cirúrgico.
Resultados. Em II espécimes cirúrgicos (37\%) o escore de Gleason foi igual ou menor que o encontrado na biópsia, enquanto em $19(63 \%)$ o escore cirúrgico foi maior que o da biópsia $(p<0,05)$. A mediana de expressão do PCNA foi, respectivamente, de $4,5 \%$, $10 \%, 12 \%$ e $14 \%$ para os escores de Gleason 2-4, 5-6, 7 e 8-10 (p> $0,05)$. A mediana dos índices de proliferação celular foi de $9 \%$ para tumores confinados à glândula ou ao espécime e de $17 \%$ para os extraprostáticos $(p<0,05)$.

Conclusão. Piora do escore de Gleason ocorreu em cerca de dois terços dos pacientes submetidos a tratamento hormonal anti-androgênico. Entretanto, os índices de proliferação celular, medidos pelo PCNA, foram iguais para espécimes com diferentes escores de Gleason. É provável que o bloqueio hormonal neo-adjuvante produza uma piora morfológica da neoplasia, sem, contudo, gerar clones celulares mais agressivos.

Unitermos: Câncer da próstata. Terapêutica antiandrogênica. Tratamento neo-adjuvante. PCNA. Imunohistoquímica.

\section{INTRODUÇÃO}

Estima-se que o risco de um homem desenvolver câncer na próstata histologicamente reconhecível durante a vida é de 42\% para norte-americanos de 50 anos de idade!. Já o risco deste tumor ser diagnosticado clinicamente é de $9,5 \%$ e a possibilidade de seu portador morrer de câncer de próstata é de 2,9\%².

O estadiamento patológico continua sendo o mais poderoso parâmetro de previsão de comportamento tumoral e do resultado do tratamento. As chances de cura dos pacientes portadores de câncer de próstata diminuem significativamente quando o estadiamento patológico indica acometimento das margens

\footnotetext{
*Correspondência:

Rua Peixoto Gomide, 2055/81

CEP: 01409-003 - São Paulo - SP

Tel: (11) 3257.8002

E-mail: srougi@terra.com.br
}

cirúrgicas, penetração no tecido periprostático, invasão das vesículas seminais e, finalmente, metástases locais ou distantes ${ }^{3}$.

Apesar do diagnóstico de câncer da próstata estar sendo realizado mais precocemente, uma grande porcentagem de pacientes já tem doença extra-capsular no momento do tratamento definitivo ${ }^{4}$. Em seis séries contemporâneas de pacientes submetidos a prostatectomia radical, o estudo anatomopatológico mostrou que a doença era órgão-confinada em somente $50 \%$ dos casos ${ }^{4-9}$.

Em virtude do mau prognóstico que envolve os pacientes com doença extracapsular ou localmente avançada, vários autores têm proposto a terapia hormonal neo-adjuvante, com o objetivo de diminuir a massa tumoral e, portanto, tornar a cirurgia radical mais efetiva $a^{4,10,11,12}$

Trabalhos utilizando análogos do $\mathrm{LH}$ $\mathrm{RH}$, isoladamente ou associado a antiandrógenos periféricos por três meses, seguido de prostatectomia radical, observaram diminuição da freqüência de margens cirúrgicas positivas para $8 \%$ a $34 \%$ em contraposição aos casos tratados somente com cirurgia, onde este fenômeno ocorreu em 33\% a 64\% 4,10-14.

Apesar dos achados cirúrgicos aparentemente favoráveis relacionados com o emprego do tratamento neo-adjuvante, notou-se que recorrência bioquímica com elevação do PSA em 2,5 anos é quase a mesma nos pacientes submetidos a terapia hormonal e naqueles submetidos somente a prostatectomia radical"!

Várias alterações histopatológicas ocorrem na próstata após a terapia hormonal neoadjuvante. A glândula sofre marcada atrofia com diminuição do seu volume, a camada de células basais torna-se proeminente, observase hiperplasia com vacuolização das mesmas e os nucléolos tornam-se menores ${ }^{12,13}$.

Mais importante: sob influência hormonal, as glândulas prostáticas apresentam-se com morfologia mais indiferenciada, que pode 
traduzir dois fenômenos distintos: deterioração fenotípica sem agravamento do comportamento biológico ou deterioração com aumento da agressividade tumora ${ }^{4,10-13}$. Esta dúvida não é apenas conceitual, mas tem implicações práticas, já que poderia explicar porquê, apesar da menor freqüência de margens positivas, observam-se as mesmas chances de progressão da doença quando se emprega ou não o tratamento hormonal neo-adjuvante associado a prostatectomia e a radioterapia.

O presente trabalho foi idealizado com o objetivo de tentar definir o potencial biológico da neoplasia em função das alterações fenotípicas que surgem nos tumores prostáticos tratados com ablação androgênica

\section{Métodos}

Foram estudados retrospectivamente 30 pacientes portadores de câncer de próstata clinicamente localizado, com idade que variou de 49 a 73 anos (mediana de 63 anos).

Após a biópsia prostática, todos os pacientes foram submetidos a estadiamento da doença através de toque retal, dosagem do PSA sérico, US transretal, tomografia computadorizada de abdômen e pélvis e cintilografia óssea.

Foram incluídos no estudo os pacientes com doença localmente avançada, ou seja, que apresentavam uma ou mais das seguintes indicações para tratamento hormonal neo-adjuvante: PSA sérico maior do que $30 \mathrm{ng} / \mathrm{ml}$ (nove pacientes), escore de Gleason maior ou igual a 7 no exame anatomopatológico da biópsia (sete pacientes), mais de $75 \%$ das biópsias positivas (seis pacientes) e estadiamento clínico $T_{2 c}$ (seis pacientes), $T_{3 a}(17$ pacientes) e $\mathrm{T}_{3 \mathrm{~b}}$ (dois pacientes). $\mathrm{O}$ tratamento hormonal foi feito com injeções mensais de análogos do LH-RH (goserelina 3,6 mg ou triptorelina 3,75 mg) por quatro meses. Os pacientes também receberam $750 \mathrm{mg}$ de flutamida oral diariamente, durante 20 dias, iniciada 10 dias antes da primeira injeção. Um mês após a quarta aplicação de análogo do LH-RH, os pacientes foram submetidos a nova dosagem do PSA sangüíneo, e, em seguida, foi realizada a prostatectomia radical retropúbica. As cirurgias de todos os pacientes foram realizadas pelo mesmo cirurgião (MS).

\begin{tabular}{|c|c|c|}
\hline \multicolumn{3}{|c|}{ Tabela I - Características iniciais da população estudada } \\
\hline Parâmetro & & Número de Pacientes (\%) \\
\hline \multicolumn{3}{|c|}{ Estadiamento } \\
\hline & Tlb & $2(6.7 \%)$ \\
\hline & Tic & $3(10.0 \%)$ \\
\hline & $\mathrm{T} 2 \mathrm{c}$ & $6(20.0 \%)$ \\
\hline & $\mathrm{T} 3 \mathrm{a}$ & $17(56.7 \%)$ \\
\hline & T3b & $2(6.7 \%)^{\prime}$ \\
\hline \multicolumn{3}{|c|}{ Escore de Gleason na Biópsia } \\
\hline & $2 a 4$ & $5(16.7 \%)$ \\
\hline & $5 a 6$ & $20(66.7 \%)$ \\
\hline & 7 a 10 & $5(16.7 \%)$ \\
\hline \multicolumn{3}{|l|}{ PSA Inicial } \\
\hline & 0 a 10 & $7(23.3 \%)$ \\
\hline & $>10$ a 20 & $10(33.3 \%)$ \\
\hline & $>20$ & $13(43.3 \%)$ \\
\hline
\end{tabular}

Gráfico I - Correlação entre a mediana de expressão do PCNA e as diferentes categorias do escore de Gleason dos espécimens cirúrgicos

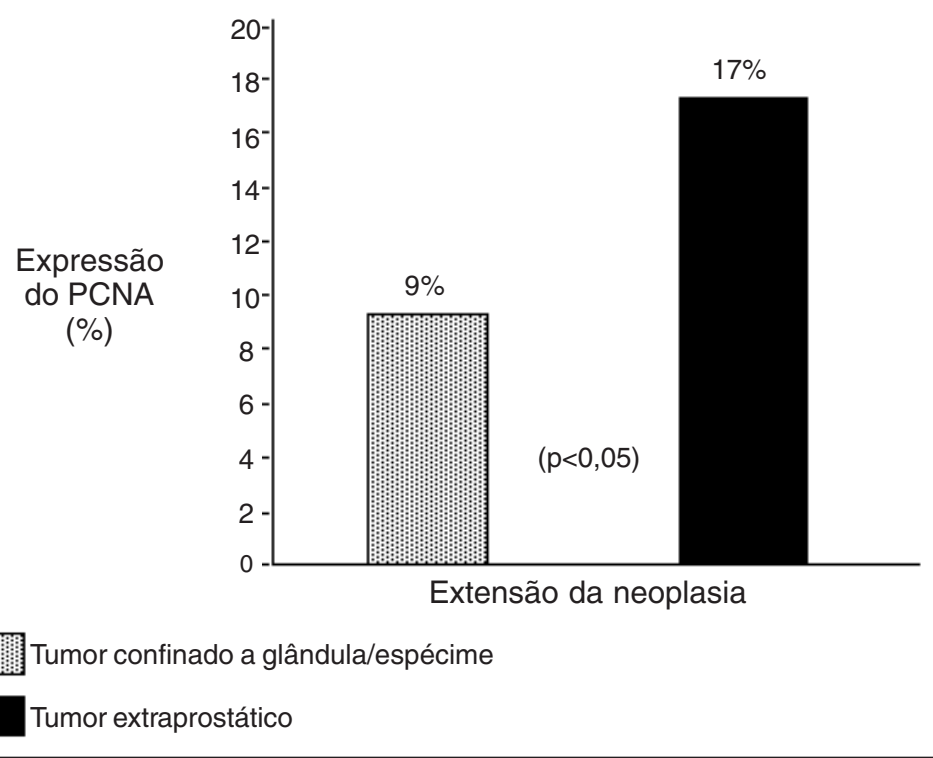

Para identificar as alterações ocorridas no tecido prostático normal e no tecido prostático neoplásico em conseqüência da terapia hormonal neo-adjuvante, foi elaborado um protocolo que incluiu as seguintes informações: a) no tecido prostático normal: atrofia glandular, proeminência das células basais, vacuolização citoplasmática, metaplasia escamosa, neoplasia intra-epitelial (PIN) de baixo e alto grau e hiperplasia adenomatosa atípica; b) no tecido neoplásico: escore de Gleason, penetração capsular, envolvimento das margens cirúrgicas, invasão vascular, infiltração do espaço perineural, invasão de vesículas seminais, vacuolização e clareamento do citoplasma, desaparecimento dos nucléolos, diminuição do tamanho do núcleo, presença de cristalóides, alterações de estroma e colabamento da luz glandular.

De modo a definir as características biológicas do tumor, recorreu-se ao emprego de método imunohistoquímico para PCNA, uma proteína nuclear associada ao ciclo celularls. Este teste permite avaliar os índices de proliferação celular, e a presença da proteína foi feita com o emprego de anticorpo monoclonal 


\section{Gráfico 2 - Correlação entre a mediana de expressão do PCNA e a extensão da neoplasia,} avaliada pelo estudo do espécime cirúrgico

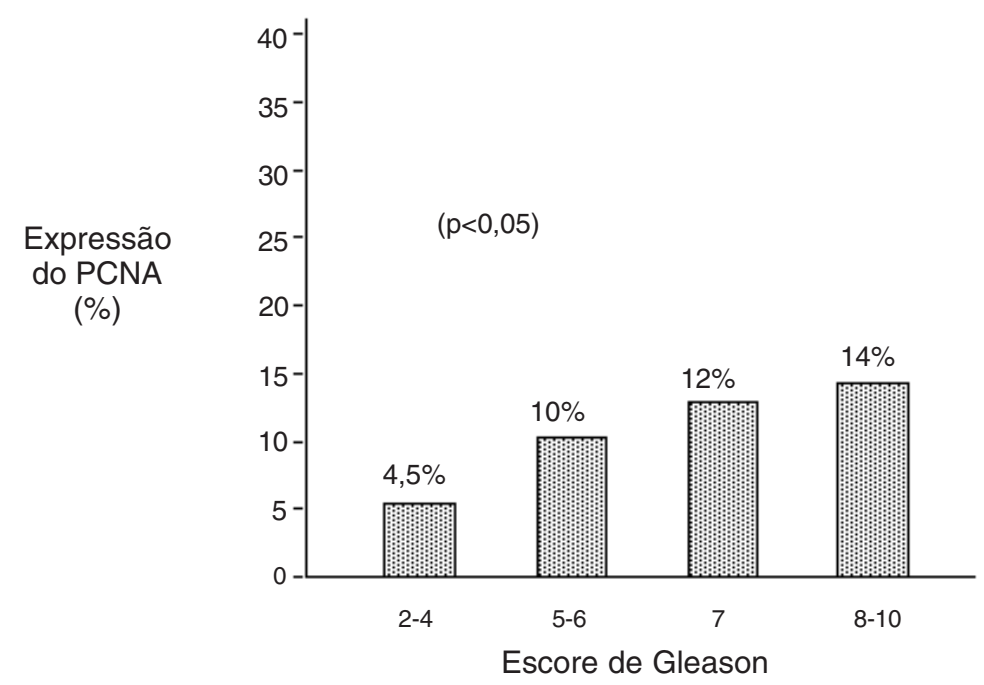

PCIO de camundongo, que reage com o PCNA (Dako, Dinamarca).

As avaliações foram realizadas com microscópio óptico pela mesma patologista (KRML). Apenas positividade nuclear intensa foi considerada positiva para o anticorpo estudado. No caso do anticorpo PCNA, pelo menos 500 células foram estudadas em área de maior positividade e a porcentagem de núcleos positivos em relação ao total contado foi definida como atividade proliferativa.

Os dois escores de Gleason (da biópsia e do espécime cirúrgico) definidos em cada paciente foram comparados, procurando-se caracterizar se houve piora ou não no grau histológico de uma fase para a outra. Foi definido como piora no escore de Gleason um aumento de dois pontos no escore ou de um ponto quando havia mudança para uma classe mais indiferenciada (escores 7 ou 8-10). Os índices de proliferação celular do tumor, estudados no espécime cirúrgico, foram correlacionados com o escore de Gleason da biópsia e da peça cirúrgica.

Para analisar as correlações entre as variáveis estudadas foram utilizados os testes de Spearman e Kendal. Para análise dos escores pareados foi empregado o teste de Wilcoxon e para as medidas das associações foi utilizado o teste do Qui-quadrado ( $\chi 2)$ e o teste de Fisher. Em todos os testes adotou-se
$5 \%(a=0,05)$ como nível crítico para rejeição da hipótese da nulidade.

\section{Resultados}

A Tabela I mostra as características iniciais da populaçãa estudada, quanto ao estádio clínico, o escore de Gleason e os níveis séricos de PSA.

Após o tratamento antiandrogênico neoadjuvante foram encontradas várias alterações no tecido neoplásico, sendo a mais freqüente - colabamento da luz das glândulas em 26 pacientes (87\%). A invasão do espaço perineural foi encontrada em 25 pacientes (83\%). O clareamento do citoplasma ocorreu em 24 pacientes (80\%) e o desaparecimento dos nucléolos com diminuição do tamanho do núcleo ocorreu em 20 pacientes (67\%). Em nove pacientes (30\%) houve coincidência ou redução do estadiamento, e em 21 casos (70\%) foi constatado um aumento no estadiamento anatomopatológico quando comparado ao estadiamento clínico.

Do total de 30 pacientes, II (37\%) apresentaram escore de Gleason após a terapia hormonal neo-adjuvante igual ou menor que o encontrado na biópsia e 19 (63\%) evidenciaram piora no escore histológico quando comparado ao da biópsia.

Quando se correlacionou a expressão do PCNA com o escore de Gleason do espécime cirúrgico (Gráfico I), observou-se a mesma mediana do PCNA nos tumores mais e menos diferenciados, respectivamente, PCNA de $4,5 \%, 10 \%, 12 \%$ e I $4 \%$ nos tumores; escore 2-4, 5-6,7 e 8-10 ( $p>0,05)$. Estes dados indicam ausência de correlação entre a piora do escore de Gleason após o tratamento neo-adjuvante e a expressão do PCNA.

A mediana da porcentagem de proliferação nuclear foi bem inferior nos casos de tumor confinado à glândula e ao espécime do que nos casos de tumor não-confinado, respectivamente $9 \%$ e $17 \%(p<0,05)$ (Gráfico 2). Estes dados demonstram que há diferença significante entre a extensão tumoral e a expressão do PCNA. Considerando-se o ponto de corte de 13,5\% para o PCNA observouse que 6 dos 18 pacientes (33\%) com tumor confinado à glândula e ao espécime e nove dos 12 pacientes (75\%) com tumor nãoconfinado apresentavam porcentagem de PCNA superior ao ponto de corte $(p<0,05)$.

\section{Discussão}

Diversos estudos retrospectivos e prospectivos demonstraram que 0 tratamento hormonal neo-adjuvante produz alterações involucionais no carcinoma prostático, diminuindo o estadiamento dos tumores localizados. Nesses trabalhos comprovou-se, também, que após a terapia de deprivação androgênica temporária, todos os pacientes persistem com

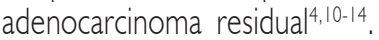

As mudanças morfológicas observadas nos casos de adenocarcinoma prostático tratados com hormônios incluem perda da arquitetura glandular, vacuolização do citoplasma e picnose nuclear. A microscopia eletrônica dos tumores tratados sugere que essas alterações involucionais são secundárias à morte celular programada ou apoptose $4,10,11,12$.

Embora a maioria desses estudos demonstre melhora no estágio da neoplasia, não está claro se esses achados se traduzem em uma melhor evolução local da doença.

Paralelamente à diminuição do estágio da doença, observa-se que o tratamento antiandrogênico produz um aumento do escore de Gleason da neoplasia, quando se comparam os achados da biópsia e do espécime cirúrgico. Este aparente aumento no escore

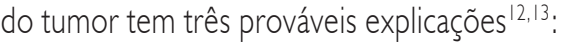
I) amostragem incompleta dos espécimes obtidos por agulha de biópsia de um tumor 
de padrão heterogêneo, com áreas de diferentes graus histológicos. 2) como resultado do encolhimento da próstata, com aparente perda da luz glandular, e do aumento do tecido periglandular. Nesta situação, o patologista seria influenciado pela falta de luz glandular e interpretaria a neoplasia como pouco diferenciada. 3) persistência dos elementos mais indiferenciados, após destruiç̧ão do componente mais favorável pelo tratamento hormonal neo-adjuvante. Este efeito poderia conferir maior agressividade à lesão remanescente, que anularia as vantagens obtidas com a redução do volume tumoral induzida pelo tratamento.

No presente estudo, observamos um aumento significativo no escore de Gleason entre os espécimes estudados antes e após a terapia hormonal, fato esse verificado em $64 \%$ dos tumores inicialmente bem e moderadamente diferenciados.

Comparando-se a expressão do PCNA em relação ao escore de Gleason do espécimes cirúrgico, observamos índices semeIhantes de atividade proliferativa nos diferentes escores de Gleason (Gráfico I). A análise destes dados indica que o aumento do escore de Gleason após a terapia hormonal neoadjuvante talvez represente um fenômeno meramente morfológico, já que a mesma atividade proliferativa nos tumores mais indiferenciados indica que a piora morfológica não se acompanha de alterações compatíveis com maior agressividade biológica das células tumorais. Por outro lado, a atividade proliferativa foi maior nos tumores extraprostáticos (Gráfico 2), o que prova que o índice de PCNA reflete o potencial de agressividade do câncer de próstata.

\section{Conclusões}

A terapia antiandrogênica neo-adjuvante nos casos de câncer da próstata produz alterações histológicas marcantes no tecido tumoral, piorando o escore de Gleason em cerca de dois terços dos casos. Contudo, os índices de proliferação celular medido pelo PCNA foram iguais para tumores com diferentes graus de diferenciação celular. Por isso, é provável que a terapia hormonal neo-adjuvante piore 0 escore de Gleason, sem que isso signifique o aparecimento de clones celulares mais agressivos, mas indique apenas uma mera alteração morfológica das células e glândulas.

\section{Conflito de interesse: não há.}

\section{SUMMARY}

Prostate cancer dedifferentiation FOLLOWING ANTIANDROGEN THERAPY: A MORPHOLOGICAL FINDING OR AN INCREASED TUMOR AGGRESSIVENESS?

BACKGROUND. Neoadjuvant androgen deprivation in prostate cancer induces tumor volume regression but does not improve outcome of the patient. A possible explanation for this phenomenon could be an increase of the residual tumor aggressiveness brought about by antiandrogen therapy. The purpose of the present study was to evaluate the frequency of tumor dedifferentiation following androgen blockade in prostate cancer and to determine if the remaining tumor shows signs of increased aggressiveness.

Methods. Thirty patients bearing locally advanced prostate cancer (stages $T_{2 c}-T_{3}$ ) were submitted to neoadjuvant anti-androgenic therapy during four months followed by radical prostatectomy. Gleason scores from biopsy and surgical specimens were compared. Furthermore, the cell proliferation index was evaluated by immunohistochemistryassay for PCNA, tests with strong nuclear staining were considered positive. The percentage of positive nuclei, counted in 500 cells, was determined in several categories of the Gleason scorefrom surgicalspecimens.

RESULTS. In I I(37\%) surgical specimens the Gleasonscorewas equal orlower than thatfound in the biopsy and in 19 (63\%) the total score was higher in the surgical specimens $(p<0.05)$. The median of PCNA expression was $4.5 \%, 10 \%$, $12 \%$ and $14 \%$ in Gleason scores 2-4, 5-6,7 and $8-10$, respectively $(p>0.05)$. The median of cell proliferation indexes was $9 \%$ for glandular or specimen confined tumors and was $17 \%$ for extraprostatic tumors $(p<0.05)$.

COnCLUsION. The lower Gleason score was found in almost 2/3 of patients submitted to antiandrogen therapy. However, the cell proliferation index measured by PCNA was the same for tumors with lower or higher Gleason scores. It seems that cell dedifferentiation seen after neoadjuvant androgen deprivation representsameremorphologicphenomenonandnota real increase in tumor aggressiveness. [Rev Assoc Med Bras 2005; 51 (2): I 17-20]

KEY WORDS: Prostate cancer. Androgen deprivation. Neoadjuvant therapy. PCNA. Immunohistochemistry.

\section{REFERÊNCIAS}

I. Scardino PT. Early detection of prostate cancer. Urol Clin North Am. 1989; 1 6: 635-655.

2. Seidman H, MushinskiMH, Gelb SK, Silverberg E. Probabilities of eventually developing or dying of cancer - United States, 1985. CA Cancer J Clin 1985; 35: 36-56.

3. Montie JE. Current prognostic factors for prostate carcinoma. Cancer 1996; 78: 34I-344.

4. Cookson MS, Sogani PC, Russo P, Herr H, Dalbagni G, Reuter VE et al. Pathological staging and biochemical recurrence after neoadjuvant androgen deprivation therapy in combination with radical prostatectomy in clinically localized prostate cancer: results of a phase II study. Br J Urol 1997; 79: 432-438.

5. Zincke H, Oesterling JE, Blute ML, Bergstralh EJ, Myers RP, Barret DM. Long-term (I5 years) results after radical prostatectomy for clinically localized (stage T2c or lower) prostate cancer. J Urol 1994; I52: I850- 1857.

6. Murphy GP, Mettlin C, Menk H, Winchester DP, Davidosn AM. National patterns of prostate cancer treatment by radical prostatectomy: results of a survey by the American College of Surgeons Commission on Cancer. J Urol 1994; 152:1917-1919.

7. Walsh PC, Partin AW, Epstein Jl. Cancer control and quality of life following anatomical radical retropubic prostatectomy: results at 10 years. J Urol 1994; |52: | 831- | 836

8. Catalona WJ, Smith DS. 5-year tumor recurrence rates after anatomical radical prostatectomy for prostate cancer. J Urol I994; 152: I837- 1842.

9. Bostwick DG, Myers RP, Oesterling JE. Staging of prostate cancer. Sem Surg Oncol 1994; 10 : 60-72.

10. Fradet $Y$. The role of neoadjuvant androgen deprivation prior to radical prostatecomy. Urol Clin North Am 1996; 23: 575-858.

I I. FairWR, Cookson MS, Stroumbakis N, Cohen D, Aprikian Ag, Wang $Y$ et al. The indications, rationale, and results of neoadjuvant androgen deprivation in the treatment of prostatic cancer: Memorial Sloan-Kettering Cancer Center results. Urology 1997; 49: 46-55.

12. Vaillancourt L, Tetû B, Fradet Y, Dupont A, Gomez J, Cusan L et al. Effect of neoadjuvant endocrine therapy (combined androgen blockade) on normal prostate and prostatic carcinoma. Am J Surg Pathol 1996; 20: 86-93.

13. Reuter VE. Pathological changes in benign and malignant prostatic tissue following androgen deprivation therapy. Urology 1997; 49: 16-22.

14. Abbas F, Kaplan M, Soloway MS. Induction androgen deprivation therapy before radical prostatectomy for prostate cancer - initial results. BrJ Urol 1996; 77: 423-428.

15. Galand P, Degraef C. Cyclin/PCNA immunostaining as an alternative to tritiated pulse labelling for markingS phase cells in parafin sections from animal and human tissue. Cell Tissue Kinet. 1989; 22:383-392.

\author{
Artigo recebido: 07/01/04 \\ Aceito para publicação: 07/06/04
}

